\title{
Influence of Ball Milling on the Local Atomic Structure of an Amorphous CoFeSiB Alloy
}

\author{
J. BednARCiK ${ }^{a}$, S. Michalik ${ }^{b}$, P. KOLlar ${ }^{b}$ AND S. Roth ${ }^{c}$ \\ ${ }^{a}$ Deutsches Elektronen-Synchrotron (DESY), Notkestr. 85, D-22607 Hamburg, Germany \\ ${ }^{b}$ Institute of Physics, P.J. Safarik University, Park Angelinum 9, 04154 Kosice, Slovakia \\ ${ }^{c}$ Institute for Metallic Materials, IFW Dresden, Helmholtzstr. 20, D-01069 Dresden, Germany \\ It follows from experimental observations that ball milling induces substantial changes in an amorphous \\ $\mathrm{Co}_{70.3} \mathrm{Fe}_{4.7} \mathrm{Si}_{10} \mathrm{~B}_{15}$ (at.\%) alloy. X-ray diffraction indicates formation of nanocrystalline bcc-(Co,Fe) phase during \\ ball milling. As a consequence crystallization sequence is altered and the total enthalpy of crystallization reduces \\ with the milling time. Furthermore thermomagnetic measurements reveal that specific magnetization and Curie \\ temperature of amorphous phase are influenced by ball milling.
}

PACS numbers: 61.43.Dq, 61.05.cp, 64.70.dg, 75.30.Kz

\section{Introduction}

Disordered nature of structural arrangement in amorphous metallic alloys gives rise to advantageous magnetic, electronic and mechanical properties. Co-based amorphous alloys with their high permeability and zero magnetostriction possess excellent soft magnetic properties that qualify them as core and sensor materials for inductive applications [1]. It was previously reported that the introduction of small fraction (less than $5 \%$ ) of nanocrystals in amorphous $\mathrm{Co}_{75} \mathrm{Si}_{10} \mathrm{~B}_{15}$ alloy refines the domain size and/or pins the domain walls, substantially reducing eddy current losses at high frequencies [2]. In this work we present the study of the partial crystallization of an amorphous $\mathrm{Co}_{70.3} \mathrm{Fe}_{4.7} \mathrm{Si}_{10} \mathrm{~B}_{15}$ alloy induced by high-energy ball milling (BM).

\section{Experimental}

Amorphous ribbons with nominal composition of $\mathrm{Co}_{70.3} \mathrm{Fe}_{4.7} \mathrm{Si}_{10} \mathrm{~B}_{15}$ (at.\%) were prepared in a form of $10 \mathrm{~mm}$ wide and about $40 \mu \mathrm{m}$ thick ribbons by single-roller melt-spinning technique. These ribbons were cut into small pieces and milled under argon atmosphere in a RETSCH PM4000 planetary ball mill up to $12 \mathrm{~h}$. BM was interrupted for every $2 \mathrm{~h}$ in order to remove small amount of sample for further investigations. Room temperature X-ray diffraction experiments utilizing high-energy photon beam $(100 \mathrm{keV})$ were performed at the wiggler beamline BW5 of the DORIS III positron storage ring at HASYLAB/DESY (Hamburg, Germany). Diffracted photons were detected using a two-dimensional imaging plate detector MAR345. Measured intensities were corrected for absorption, fluorescence, multiple and Compton scattering. Structure factor was calculated using standard procedures described in [3]. The thermal stability of the melt-spun ribbons and the ball-milled powders was examined at a heating rate of $10^{\circ} \mathrm{C} / \mathrm{min}$ by differential scanning calorimetry (DSC) using a NETZSCH DSC 404. The Curie temperature of amorphous phase $T_{\mathrm{C}}$ was determined from the first derivative of the thermomagnetic $M(T)$ curves traced at $10^{\circ} \mathrm{C} /$ min using a Faraday magnetic balance.

\section{Results and discussion}

The evolution of the total structure factors $S(q)$ with the milling time $t_{\mathrm{m}}$ is shown in Fig. 1. At first sight $S(q)$ 's look rather similar, however careful comparison indicates slight changes visible on ball-milled samples. Plotting the difference structure factors with respect to the as quenched alloy, for example $S(q)_{6 \mathrm{~h}}-S(q)_{\text {aq }}$ (see dashed line in Fig. 1), indicates the presence of relatively sharp peaks belonging to crystalline phase. From the positions of these maxima it seems they are stemming from cubic bcc- $(\mathrm{Co}, \mathrm{Fe})$ phase with the lattice parameter $2.866 \AA$. It is interesting to note that intensity of the Bragg peaks increases during the early stages (up to $4 \mathrm{~h}$ ) of the BM whereas longer milling leads into significant broadening and weakening of the Bragg peaks. To observe structural changes in a real space, obtained structure factors $S(q)$ were Fourier transformed thus yielding reduced radial distribution function $G(r)$ defined by equation:

$$
\begin{aligned}
G(r) & =4 \pi r\left[\rho(r)-\rho_{0}\right] \\
= & \frac{2}{\pi} \int_{0}^{q_{\max }} q[S(q)-1] \sin (q r) \mathrm{d} q,
\end{aligned}
$$

in which $q$ is the momentum wave vector transfer, $r$ denotes radial distance, $\rho(r)$ and $\rho_{0}$ represent local and mean atomic density, respectively. As can be seen from the inset in Fig. 1, $\mathrm{Co}-\mathrm{Co}, \mathrm{Co}-\mathrm{Fe}, \mathrm{Co}-\mathrm{Si}$ and $\mathrm{Co}-\mathrm{B}$ are the most significant atomic partials which determine the shape of the first coordination sphere. The changes in the local atomic structure induced by BM are manifested by an increase of the intensity and shift of the first coordination shell towards lower distances for the sample milled for $12 \mathrm{~h}$.

Even more pronounced changes are revealed by DSC measurements which are presented in Fig. 2. The as quenched alloy exhibits glass transition $\left(T_{\mathrm{g}}=550^{\circ} \mathrm{C}\right)$ followed by a two-stage crystallization $\left(T_{x 1}=575^{\circ} \mathrm{C}\right.$, 


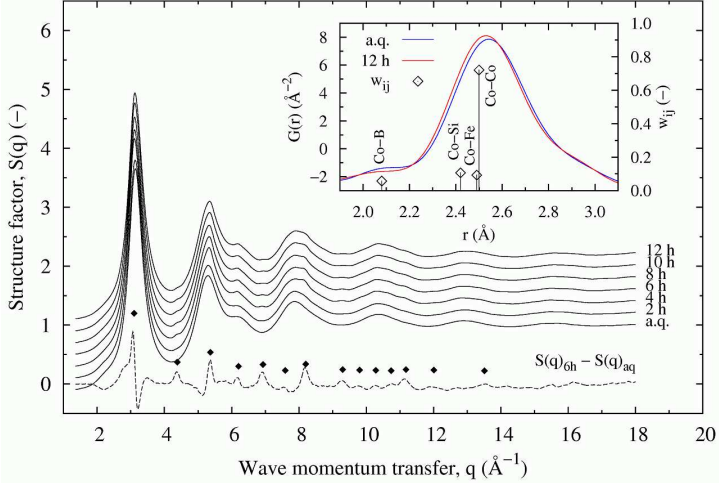

Fig. 1. Evolution of the structure factors $S(q)$ of $\mathrm{Co}_{70.3} \mathrm{Fe}_{4.7} \mathrm{Si}_{10} \mathrm{~B}_{15}$ alloy with the milling time $t_{\mathrm{m}}$. Peaks appearing on the difference structure factor $S(q)_{6 \mathrm{~h}^{-}}$ $S(q)_{\text {aq }}$ (magnified 5 times) belong to bcc- $(\mathrm{Co}, \mathrm{Fe})$ cubic phase (marked by filled diamonds). The inset shows detailed view of the reduced pair distribution function $G(r)$ depicting the first coordination shell together with the most significant $\left(w_{i j}(q=0)>0.05\right)$ atomic partials based on the sum of the nominal atomic radii.

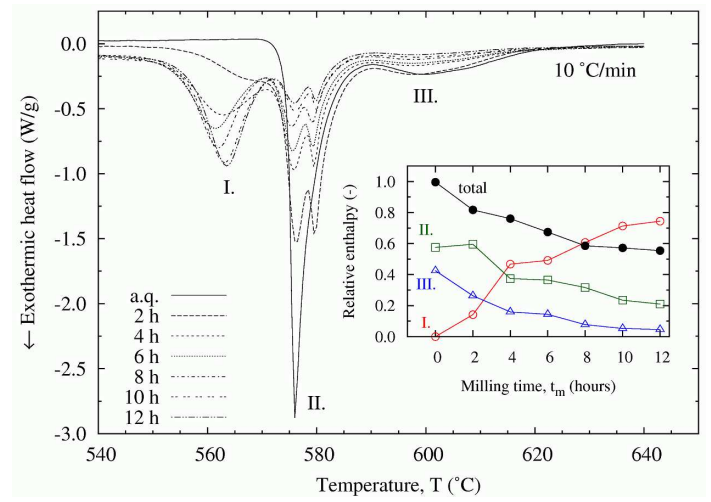

Fig. 2. DSC curves of $\mathrm{Co}_{70 .} \mathrm{Fe}_{4.7} \mathrm{Si}_{10} \mathrm{~B}_{15}$ alloy at different stages of ball milling. The inset shows behaviour of the relative crystallization enthalpy associated with the first (I), second (II) and the third (III) exothermic events, respectively, at a given milling time. The curve with filled circles shows relative (with respect to as quenched alloy) decrease of the total crystallization enthalpy with the milling time $t_{\mathrm{m}}$.

$\left.T_{x 2}=590^{\circ} \mathrm{C}\right)$. On the other hand, no glass transition is observed in a case of ball-milled samples. The BM induces such structural changes so that two-step crystallization transforms to the three-step event (labelled by I, II and III in Fig. 2) by formation of an additional exothermic peak (I) located in the former supercooled liquid region. As can be seen from the inset in Fig. 2, the enthalpy of the crystallization associated with the exothermic peak I increases with the BM time whereas enthalpies associated with the exothermic peaks II and III decrease. Since the total crystallization enthalpy decreases with the $\mathrm{BM}$ time, one may conclude that the nanocrystalline cubic bcc- $(\mathrm{Co}, \mathrm{Fe})$ phase observed by XRD is most probably a result of mechanically induced crystallization rather than contamination from milling media [4].
This is also supported by thermomagnetic measurements on powder samples showing increase of the room temperature magnetization and shift of the Curie temperature with the milling time (see Fig. 3). We suppose partial crystallization of $\mathrm{CoFeSiB}$ alloy induced by $\mathrm{BM}$ leads to formation of bcc- $(\mathrm{Co}, \mathrm{Fe})$ which in turn may cause the redistribution of atoms and formation of clusters inside amorphous phase [5]. One could conclude that $T_{\mathrm{C} 2}$ observed for powders milled longer than $6 \mathrm{~h}$ corresponds to those clusters.

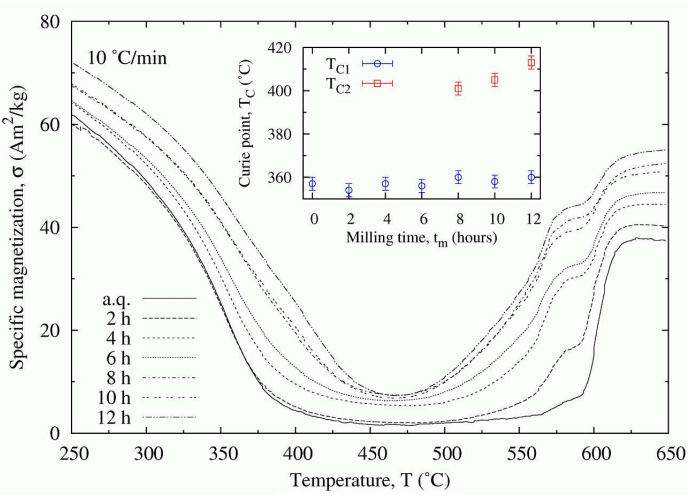

Fig. 3. Thermomagnetic curves of $\mathrm{Co}_{70.3} \mathrm{Fe}_{4.7} \mathrm{Si}_{10} \mathrm{~B}_{15}$ as quenched ribbon and ball-milled powders. The inset shows dependence of the Curie point $T_{\mathrm{C}}$ on the milling time $t_{\mathrm{m}}$.

\section{Conclusion}

The observed changes in thermal stability, crystallization behaviour, specific magnetisation and Curie temperature upon milling reflect strong influence of BM on microstructure of milled $\mathrm{Co}_{70 .} \mathrm{Fe}_{4.7} \mathrm{Si}_{10} \mathrm{~B}_{15}$ alloy. Furthermore, our structural investigations show that BM represents an alternative way to partially devitrify amorphous $\mathrm{Co}_{70.3} \mathrm{Fe}_{4.7} \mathrm{Si}_{10} \mathrm{~B}_{15}$ alloy and thus demonstrate that nanocomposite (bcc-(Co,Fe) nanocrystals embedded in amorphous matrix) material may be prepared in an easily controllable way.

\section{Acknowledgments}

The work presented in this paper was supported by the Slovak Grant Agency VEGA grants no. 1/0311/10 and no. $1 / 0167 / 10$.

\section{References}

[1] M.E. McHenry, M.A. Willard, D.E. Laughlin, Prog. Mater. Sci. 44, 291 (1999).

[2] A.K. Shaikh, D. Wexler, G.W. Delamore, J. Magn. Magn. Mater. 152, 345 (1996).

[3] T. Egami, S.J.L. Billinge, Underneath the Bragg Peaks: Structural Analysis of Complex Materials, Pergamon Press, Elsevier, Oxford 2003.

[4] J. Bednarcik, E. Burkel, E. Saksl, P. Kollar, S. Roth, J. Appl. Phys. 100, 014903 (2006).

[5] C. Miguel, S. Kaloshkin, J. Gonzalez, A. Zhukov, J. Non-Cryst. Solids 329, 63 (2003). 